\title{
Schwannoma: Differential diagnosis of a subepithelial gastric lesion. Two case report
}

\author{
Schwannoma: diagnóstico diferencial de lesiones subepiteliales gástricas. Reporte de \\ dos casos
}

\author{
Mikel Rojo-Abecia*, Leyre López-Antoñanzas, Rocío B. Avellana-Moreno, Enrique Camarero-Rodríguez, \\ Eva García-Romero, Pablo Talavera-Eguizabal, and Antonio J. Torres-García \\ General Surgery and Digestive System Service, Hospital Clínico San Carlos, Madrid, Spain
}

\begin{abstract}
The pre-operative differential diagnosis of gastric subepithelial lesions is complex. We can find pathologies with a very different behavior. Some of them, like gastrointestinal (GI) stromal tumors, can present a malignant behavior, and others like schwannomas are practically benign. Schwannomas of the GI tract originate from the Schwann cells of the Auerbach plexus and their most frequent location is the stomach. The definitive diagnosis is made by immunohistochemical analysis of the surgical specimen and its resection is curative. We report two cases of gastric subepithelial lesions with a definitive diagnosis of schwannoma.
\end{abstract}

Key words: Gastric schwannoma. Subepithelial gastric lesion. Gastrointestinal stromal tumors.

\section{Resumen}

El diagnóstico diferencial preoperatorio de las lesiones subepiteliales gástricas es complejo. Podemos encontrar patologías con un comportamiento muy diferente. Algunas de ellas, como los GIST (gastrointestinal stromal tumours), pueden presentar un comportamiento maligno, y otras, como los schwannomas, son prácticamente benignas. Los schwannomas del tracto gastrointestinal se originan de las células de Schwann del plexo de Auerbach y su localización más frecuente es el estómago. El diagnóstico definitivo se realiza mediante el análisis inmunohistoquímico de la pieza quirúrgica, y su resección es curativa. Reportamos dos casos de lesiones subepiteliales gástricas con diagnóstico definitivo de schwannoma.

Palabras clave: Schwannoma gástrico. Lesión subepitelial gástrica. GIST.

\section{Introduction}

Gastrointestinal (GI) schwannomas origin from Schwann cells sheath of the Auerbach's plexus'. They are classified as mesenchymal tumors such as leiomyomas, Gl stromal tumors (GIST), lipomas, and hemangiomas².
Schwannomas represent 2-6\% of GI mesenchymal tumors $^{3}$. The most common localization is the stomach, followed by the colon and the rectum ${ }^{4}$.

Gastric schwannomas are almost always detected as a subepithelial mass like other mesenchymal tumors of the stomach ${ }^{5}$. Although they have a similar presentation, they have different behavior, therapeutic options,

Correspondence:

*Mikel Rojo-Abecia

San Bernardo, 115 


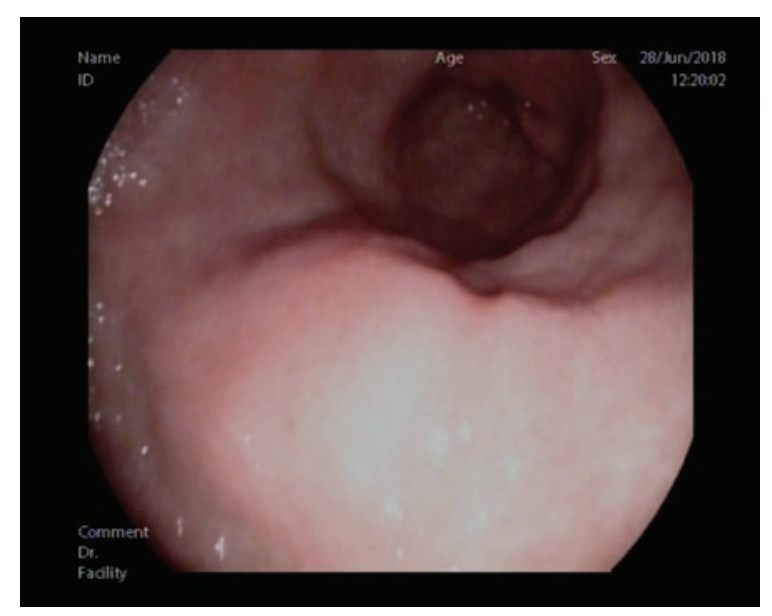

Figure 1. Gastroscopy of the first case where a $2 \mathrm{~cm}$ elevated lesion with respected mucosa is observed.

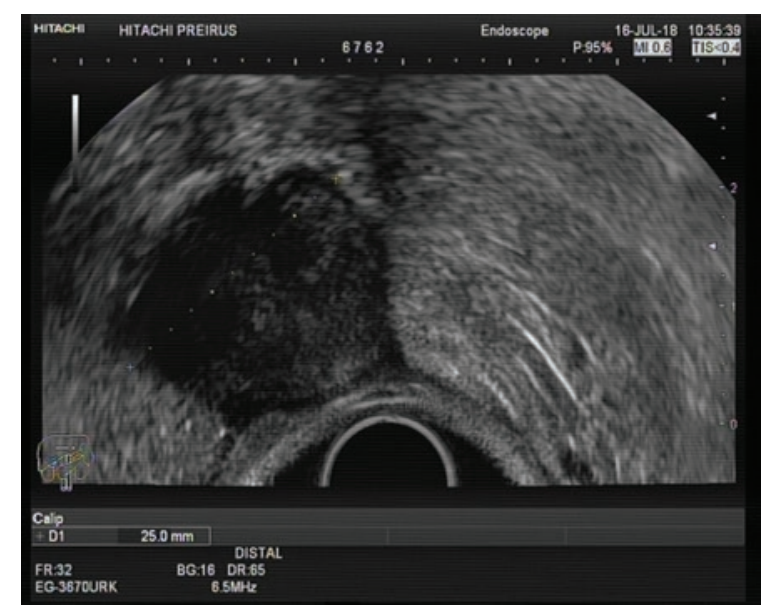

Figure 2. Endoscopic ultrasound of the first case showing a $26 \mathrm{~mm}$ lesion, hypoecogenic and dependent on the $4^{\text {th }}$ layer.

and prognoses. What makes each mesenchymal tumor different are the specific histology and immunohistochemical markers.

\section{Clinical cases}

The first case is a 66-year-old female with obesity, type 2 diabetes, hypertension, and atrial fibrillation. She was admitted to the hospital for dysphagia of 6 months of evolution. The patient reports progressive worsening, with intolerance to solid food. Within the diagnostic study, an ultrasound of the abdomen was performed, showing a solid lesion in the wall of the gastric antrum. In view of this finding, a high-resolution gastroscopy was performed. The examination showed

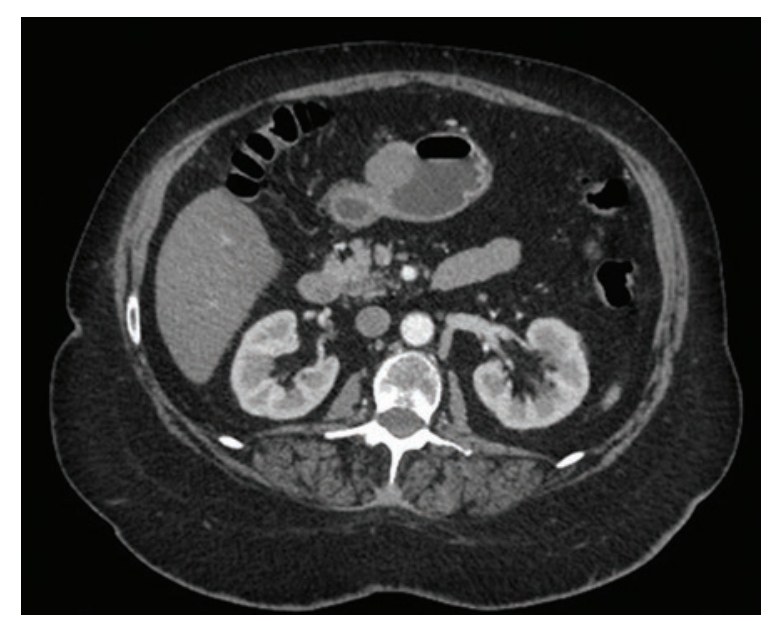

Figure 3. Computed tomography scan with a $3 \mathrm{~cm}$ exophytic mass in the antrum.

a $5 \mathrm{~cm}$ hiatal hernia and an elevated $20 \mathrm{~mm}$ lesion in the major curvature covered with normal mucosa, suggestive of extrinsic compression (Fig. 1). Endoscopic ultrasound showed a hypo-echoic and heterogeneous $26 \mathrm{~mm}$ lesion suggestive of GIST in the gastric body. This lesion was in the $4^{\text {th }}$ layer, the muscularis propia (Fig. 2). The abdominal computed tomography (CT) evidenced a $3 \mathrm{~cm}$ exophytic mass in the antrum (Fig. 3). With the suspicion of a gastric GIST, a laparoscopic resection of the mass was performed. During the same procedure, assuming that the hiatal hernia could be the cause of the dysphagia, a hiatoplasty with mesh and a Nissen-type fundoplication was performed. The piece showed an intraluminal tumor of $3 \times 2.5 \mathrm{~cm}$ with an elastic consistency and a lobed surface that respects the mucosa and retracts the serosa. Under the microscope, a neoplastic mesenchymal proliferation was observed, centered on the muscular layer, respecting the mucosa, submucosal, and serosa. It was constituted by spindle cells arranged in fascicles with collagen stroma and without necrosis. No mitosis figures were observed. The immunohistochemical study presented a tumor with positive staining to $\mathrm{S}-100$ and negative to CD34, CD 117, actin, and desmin, a typical immunohistochemistry of schwannoma (Fig. 4). After 18 months of follow-up, the patient showed no signs of tumor recurrence in the complementary control tests. In addition, dysphagia disappeared after surgery.

The second case is about a 64-year-old male who was followed for 9 years for a subepithelial gastric mass. He was diagnosed with an magnetic resonance imaging (MRI) performed on suspicion of choledocholithiasis. Initially, the lesion, located in the minor curvature of the 


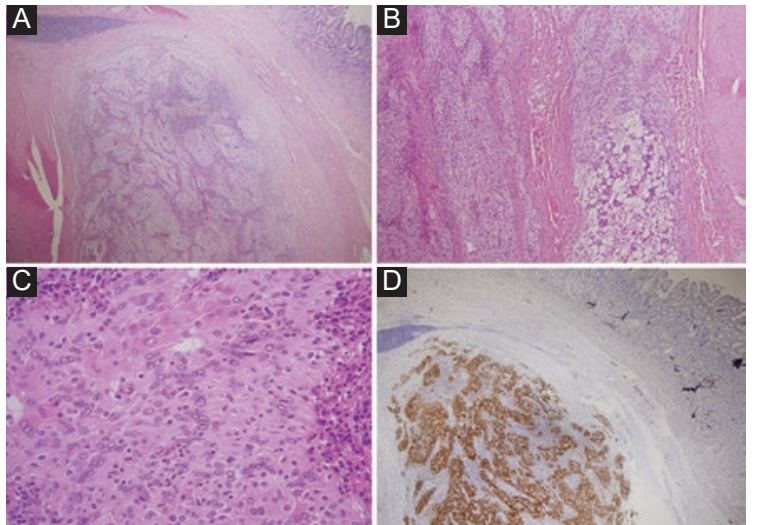

Figure 4. Immunohistochemistry of the first patient's tumor. A: wavy splindled cells separated by woven fibrotic bundles. (H-E 10X). B: gastric schwannoma shows spindle cel tumors (H-E 10x). C: the tumors cells are spindle shapped with elongated nuclei and form loose interlacing fascicles or whorls (H-E 40X). D: intense expression of S-100 immunostain in tumor cells.

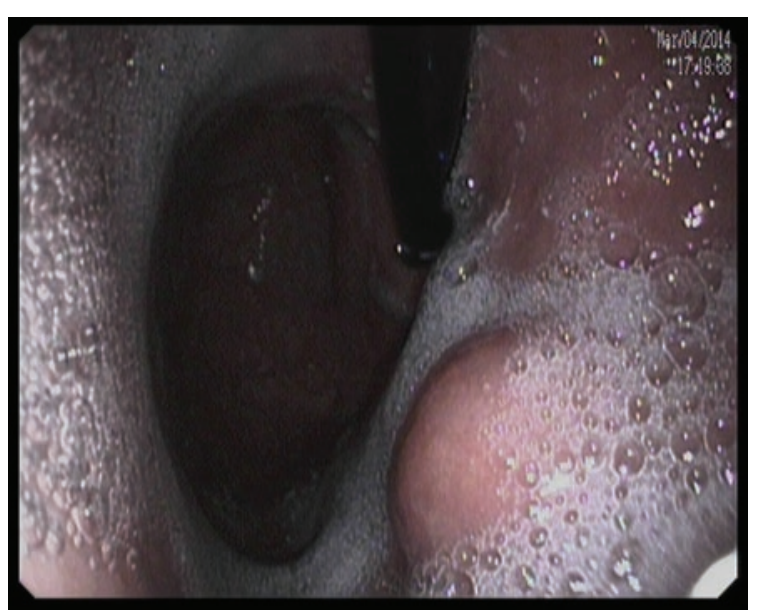

Figure 5. Gastroscopy of the second case where a $4 \mathrm{~cm}$ elevated lesion covered with normal mucosa.

gastric body, was $15 \mathrm{~mm}$ in diameter. In subsequent controls, it increased in size. In the last CT scan, the size was $30 \mathrm{~mm}$. The high-resolution endoscopy showed an oval lesion of about $4 \mathrm{~cm}$ in the anterior face of the gastric body covered with normal mucosa (Fig. 5). Endoscopic ultrasound showed a slightly heterogeneous and hypoechoic lesion of $2.5 \times 1.5 \mathrm{~cm}$ in diameter in the upper gastric body. The study showed that the lesion was located in the $4^{\text {th }}$ layer (Fig. 6). In view of the progressive growth, it was decided to perform a laparoscopic resection of the tumor. The anatomopathological study showed a $2 \times 2.5 \mathrm{~cm}$ intramural node that respects the mucosa and serosa. Microscopically, it was a neoplastic fusocellular proliferation centered in the muscular

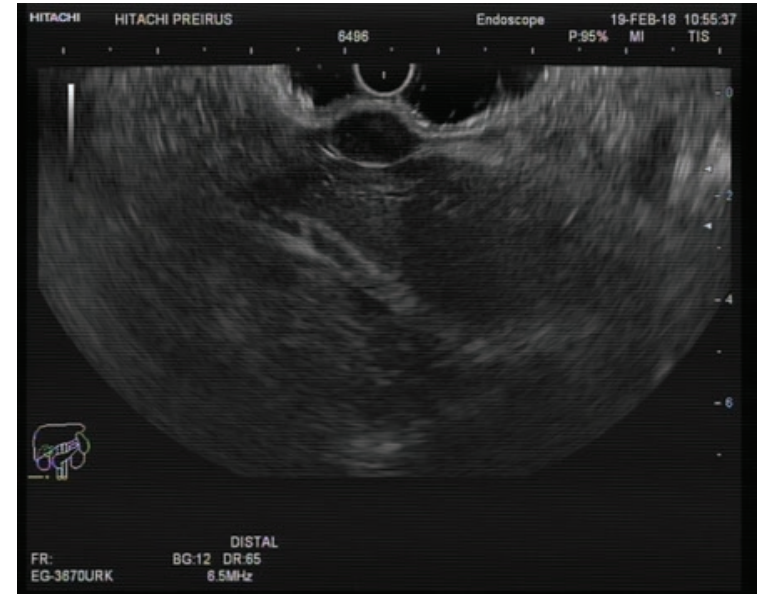

Figure 6. Endoscopic ultrasound of the second case showing a slightly heterogeneous and hypoechoic lesion of $2.5 \times 1.5 \mathrm{~cm}$ located in the $4^{\text {th }}$ layer.
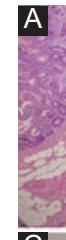

\section{C}

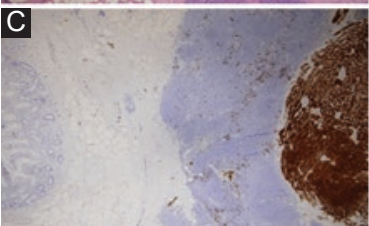

Figure 7. Immunohistochemistry of the second case tumor. A: gastric wall with intramuscular lesion, with prominent reactive lymphoid infiltrate in the periphery $(H-E 4 X)$. B: fusiform cells without cytologic atypia and significant mitosis (H-E 40x). C and D: intense expression of S-100 immunostain in tumor cells.

layer with collagen stroma and without necrosis. The cells presented a poorly defined eosinophilic cytoplasm, nuclear atypia, and low mitotic index (1 mitosis per 50 high magnification fields). Immunohistochemistry showed intense positivity to S-100 and negativity to CD34, CD117, actin, and desmin (Fig. 7). After 2 years of follow-up, there is no evidence of recurrence after a gastroscopy and a CT scan of the abdomen.

\section{Discussion}

Gastric subepithelial tumors are found in approximately 1 in 300 gastroscopies performed on adults. They occur as protrusions on the inner surface of the gastric lumen covered by normal epithelium. These 


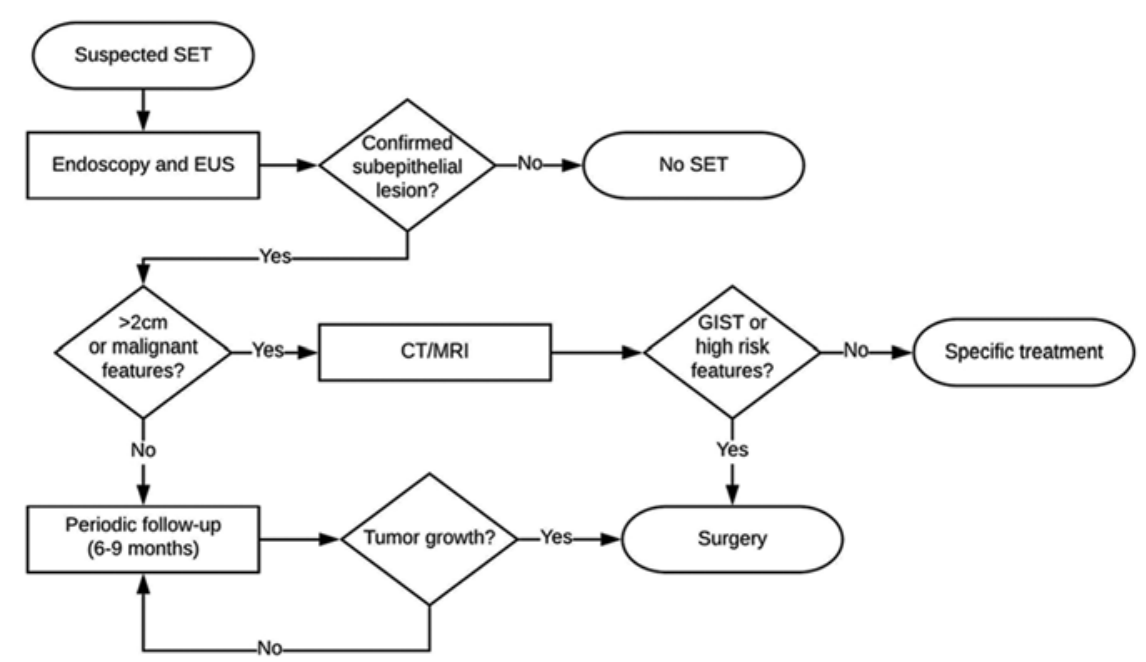

Figure 8. Protocol for the management of asymptomatic subepithelial gastric tumors in our institution.

lesions may be caused by a tumor in the gastric wall or by extrinsic compression.

These tumors are classified as non-neoplastic and neoplastic. The non-neoplastic ones include inflammatory lesions, cysts, and ectopic pancreas. The most important neoplastic lesions are mesenchymal tumors and lymphomas. Mesenchymal tumors mainly include GIST, myogenic tumors such as leiomyomas and leiomyosarcomas, neurogenic tumors including schwannoma and neurofibroma and vascular tumors ${ }^{7}$.

In this paper, we present two cases of schwannomas. These tumors originate from the Schwann cells of the nerve sheaths. They are rare in the GI tract and their most frequent location is the stomach followed by the rectum. Their clinical presentation is variable, but most are asymptomatic, and their diagnosis is casual. The symptoms, when present, are usually due to their complications (digestive bleeding, compression, obstructive symptoms due to abdominal mass, and invagination) ${ }^{8}$. In endoscopy, they present as subepithelial elevated lesions with a firm consistency. Endoscopic ultrasound usually shows a hypoecogenic lesion originating from the $4^{\text {th }}$ layer (muscle layer) $^{9}$. Contrast-enhanced CT shows homogeneous attenuation with minimal increase in the arterial phase and delayed increase in the equilibrium phase ${ }^{7}$.

Due to its frequency and potential for malignancy, the main differential diagnoses of gastric schwannomas are GISTs. Their most frequent location is the stomach. The most frequent symptoms are bleeding and abdominal discomfort due to the mass effect.
Some studies show that between 15 and $30 \%$ are asymptomatic $^{10}$. The endoscopic features of GISTs are common to all other gastric subepithelial tumors, a smooth bulge covered by normal mucosa. When these tumors grow, they may show mucosal ulceration. Endoscopic ultrasound usually shows a hypochoic or anechoic and slightly heterogeneous tumor. Hyperecogenicity can be a sign of malignancy. The tumor usually depends on the $3^{\text {rd }}$ or $4^{\text {th }}$ layer and rarely on the $2^{\text {nd }}$ layer. CT images vary according to size and aggressiveness. Small and benign lesions are usually well-defined, homogeneous with variable contrast enhancement. Large and aggressive tumors may be lobulated, with irregular margins, mucosal ulceration, central necrosis, and heterogeneous contrast enhancement?

There are methods for sampling these tumors to make a histological diagnosis based on endoscopy or endoscopic ultrasound ${ }^{11}$. However, if the suspected diagnosis is a GIST, due to the risk of bleeding, we do not perform these kinds of tests in our hospital. Therefore, we apply the following protocol (Fig. 8): first, an endoscopy is performed to exclude epithelial lesions. Then, an endoscopic ultrasound confirms that the lesion is subepithelial. If the lesion is $<2 \mathrm{~cm}$ and shows no suspicious signs, only follow-up is performed. If it is larger than $2 \mathrm{~cm}$ or has any suspicious features, the next step is to perform a CT scan or MRI to better characterize the lesion and its possible dissemination. If, after these studies, the suspected lesion is GIST or another lesion suggestive of malignancy, the lesion will 
be surgically removed if there is no contraindication for it.

This protocol applies to casually diagnosed subepithelial tumors. If the tumor produces symptoms, and there is no contraindication, treatment would be surgical. The therapeutic algorithm is based on the National Comprehensive Cancer Network guidelines which indicate that tumors suspected of GIST larger than $2 \mathrm{~cm}$ or those with high-risk characteristics are more likely to behave malignantly, and therefore, treatment should be surgical. If the suspicion is clearly of another type of lesion, the taking of samples for diagnosis could be considered, and the corresponding treatment applied.

This therapeutic management implies that the differential diagnosis of these tumors is generally done post-operatively in our institution. This diagnosis is based on the immunohistochemical examination of the surgical pieces. Schwannomas are positive for S-100 and negative for CD117, CD34, SMA, and des$\mathrm{min}$. Performing this diagnosis properly is very important because, in contrast to GIST, they are tumors with a very low malignant potential and resection with margins is practically curative.

\section{Conclusion}

Pre-operative differential diagnosis of gastric subepithelial lesions is complex. Endoscopy and endoscopic ultrasonography are often non-specific and are unable to differentiate practically benign lesions such as schwannomas from potentially malignant lesions like GIST. In our center, the therapeutic management is not based on a histological diagnosis due to the risk of bleeding from GIST after a puncture and is based on imaging characteristics and size. Therefore, the definitive diagnosis is made after the immunohistochemical analysis of the piece. This diagnosis is very important because resection with margins is practically curative in the case of schwannomas.

\section{Conflicts of interest}

The authors declare that they have no conflicts of interest and have not received external sources of funding.

\section{Ethical disclosures}

Protection of human and animal subjects. The authors declare that no experiments were performed on humans or animals for this study.

Confidentiality of data. The authors declare that they have followed the protocols of their work center on the publication of patient data.

Right to privacy and informed consent. The authors have obtained the written informed consent of the patients or subjects mentioned in the article. The corresponding author is in possession of this document.

\section{References}

1. Lin CS, Hsu HS, Tsai CH, Li WY, Huang MH. Gastric schwannoma. J Chin Med Assoc. 2004;67:583-6.

2. Al-Harake A, Chour M, Al Beteddini OS. Solitary intestinal neurofibroma with no associated systemic syndromes causing intussusception: case report and literature review. Int J Surg Case Rep. 2013;4:629-32.

3. Hou YY, Tan YS, Xu JF, Wang XN, Lu SH, Ji Y, et al. Schwannoma of the gastrointestinal tract: a clinicopathological, immunohistochemical and ultrastructural study of 33 cases. Histopathology. 2006;48:536-45

4. Oh SJ, Suh BJ, Park JK. Gastric schwannoma mimicking malignant gastrointestinal stromal tumor exhibiting increased fluorodeoxyglucose uptake. Case Rep Oncol. 2016:9:228-34.

5. Levy AD, Quiles AM, Miettinen M, Sobin SH. Gastrointestinal schwannomas: CT features with clinicopathologic correlation. AJR Am J Roentgenol. 2005;184:797-802.

6. Papanikolaou IS, Triantafyllou K, Kourikou A, Rosch T. Endoscopic ultrasonography for gastric submucosal lesions. World J Gastrointest Endosc. 2011;3:86-94.

7. Nishida T, Kawai N, Yamaguchi S, Nishida Y. Submucosal tumors: comprehensive guide for the diagnosis and therapy of gastrointestinal submucosal tumors. Dig Endosc. 2013;25:479-89.

8. Bosolino A, De la Torre A, Ratto R, Marzano C. Schwannoma gástrico. Gastroenterol Hepatol. 2010;33:686-7.

9. Hu J, Liu X, Ge N, Wang S, Guo J, Wang G, et al. Role of endoscopic ultrasound and endoscopic resection for the treatment of gastric schwannoma. Medicine (Baltimore). 2017;96:e7175.

10. Akahoshi K, Oya M, Koga T, Shiratsuchi Y. Current clinical management of gastrointestinal stromal tumor. World J Gastroenterol. 2018;24:2806-17.

11. Johns E, Binmoeller KF. Subepithelial lesions: a deeper look. Gastrointest Endosc. 2016;84:930-2. 